\title{
Improving the surgical hot clinic
}

\author{
Thomas Hubbard, Rhys Thomas
}

Croydon University Hospital, London

\begin{abstract}
Ambulatory care is an underdeveloped concept in the setting of emergency surgery, however it is recognised that many institutions will need to develop this service to cope with increased time and financial pressures.[1] There is increased emphasis on ambulatory care pathways for a variety of medical conditions.[2] Risk management is important in managing patients with acute abdominal pain in an outpatient setting and senior doctor support is essential. While the patient remains in the community, effective communication with the patient's primary care provider improves patient safety and satisfaction.[3]

This quality improvement project identified current service provision of ambulatory care for surgical patients in the hot clinic at Croydon University Hospital with subsequent consultation with the surgical department to identify problems arising from the throughput of patients. Guidelines were then updated incorporating solutions to the identified issues which were then validated by the department of general surgery. Post intervention measurement identified a decrease in patients whose principal assessment and management was made by a senior house officer level doctor through the hot clinic patient journey from $26 \%$ to $9 \%$ (64\% decrease), indicating an increase in registrar and/or consultant involvement in managing the hot clinic. The number of patients attending hot clinic that had effective discharge liaison (in the form of a formal letter) to the GP increased from $18 \%$ to $68 \%$ (250\% increase). In conclusion, the introduction of updated guidelines effected a safer and more effective ambulatory hot clinic to perform closer to full capacity, providing improved patient care for the local population.
\end{abstract}

\section{Problem}

Croydon University Hospital is a large district general hospital in Southwest London providing care to a mixed local population of over 350,000 people. The hospital has an established general surgical ambulatory "hot clinic". The service is managed by an senior house officer level junior trainee who is responsible for the assessment and management of these patients under the close supervision of the on-call registrar and consultant. Hot clinic patients will have been assessed by the emergency general surgical team, usually in the preceding 24 hours, and identified as suitable for ambulatory care. The patient attends hot clinic either for a clinical review or an ultrasound scan (of which there are two slots every day Monday to Friday for use by solely by the hot clinic patients).

A disparity between Royal College of Surgeons ambulatory clinic guidelines and local guidelines was identified which implied the current hot clinic model did not reflect the best standard of care. Two principal issues identified from the new guidelines that this quality improvement project was designed to address in local practice:

1. Patients were often managed by Foundation Year 2 or SHOlevel doctors in the ambulatory clinic, frequently with no senior input

2. Patients had investigations and management plans formulated in hot clinic with poor or inconsistent communication with their primary care provider.

Although no clinical incidents, "never events", or untoward incidents were identified, these issues were thought to lead to sub-optimal care of patients in the hot clinic with an unacceptably high risk of future incidents.

\section{Background}

Emergency surgery for conditions such as peritonitis, obstruction, and other abdominal catastrophes is a high risk and high costly area of surgical practice requiring in-patient management. There are a wide variety of other conditions managed by the acute surgical team that are less acute but classically result in an expensive inpatient stay, usually for diagnostic tests and which often do not result in definitive in-patient management. Examples of these conditions are cholecystitis, biliary colic, mild uncomplicated diverticulitis, and non-specific abdominal pain. Royal college of Surgeons of England guidelines outlines how these conditions may be safely managed in an ambulatory setting,[1] reducing costs to the healthcare provider and the inpatient burden to the acute surgical team. Valuable time can therefore be released for the assessment and management of acutely unwell patients requiring inpatient management. Ambulatory care is well developed in medicine,[2] with most departments in the United Kingdom offering a service for a range of medical conditions. However, this service is much less developed in the surgical setting, with a limited evidence base.

This premise was explored and reported on recently, with new guidelines being drafted by the Royal College of Surgeons commissioning guide: emergency general surgery (acute abdominal pain). The guidelines focus on a number of areas, but this QIP focuses on section 1.3, Emergency surgery ambulatory care (ESAC) pathway. The RCS predicts that up to $30 \%$ of patients on an acute general surgical take could be managed using this 
pathway and thus avoid hospital admission.

Underpinning the expansion and improvement of the ambulatory service is risk management. Patients with acute abdominal pain have a variety of differential diagnosis ranging from the benign to the immediately life threatening. Therefore a patient must be adequately assessed with appropriate senior input, and if managed as an outpatient, communication with the patient's primary care provider (GP) is essential for continuity of care and to ensure patient satisfaction.[3]

\section{Baseline measurement}

A baseline retrospective audit was performed to measure the scale of the problem. All patients attending the hot clinic between 1st May and 30th June 2014 were included $(n=69)$.

Eligible patients were identified through the daily register of patients attending hot clinic that are kept in the ward where patients attend, this was cross checked against the daily "take" lists that are kept by the on call senior house officer (SHO) that are saved to the local hard drive.

Patient notes were accessed through the local electronic notes system, where all results, observations, and discharge scripts are available.

The following measurements were made:

- Grade of doctor assessing in $\mathrm{A}+\mathrm{E}$

- Whether patient was discussed with senior

- Initial diagnosis, grade of doctor assessing in hot clinic

- Whether patient had a discharge letter sent to GP.

All calculations and graphs were made using Microsoft Excel.

Results of this baseline measurement showed that:

- $44 \%$ of patients were seen in $A+E$ by an $\mathrm{SHO}$ only without discussion with a senior

- Of these $44 \%, 58 \%$ were then assessed in hot clinic by an $\mathrm{SHO}$ only without senior discussion

- Therefore $26 \%$ of patients going through the hot clinic pathway did not have senior input during their visit to the hospital and ambulatory clinic

- $18 \%$ of patients attending hot clinic had a letter sent to primary care provider.

\section{Design}

The final intervention was to change the local guidelines for the hot clinic to reflect the RCS guidelines, and improve patient care. This would be a sustainable change in the long term as all new doctors to the department have access to the guidelines on the Trust intranet.

For this to be an effective and sustainable change the whole department would be consulted at various stages throughout the project for a department wide agreement on the changes. The greatest barrier to improving the service would be changing attitudes towards the service, thus the project required support from every grade of doctor in the department from FY1 to consultant. Presenting the initial audit and highlighting two key areas of improvement (senior support and GP communication), the consultants were keen to change the department's attitudes to the service.

Raising awareness became an essential part of the project. At each stage of the project, discussion was encouraged to create a service centred around improving patient care while also providing the evolution of realistically achievable goals for the department to meet. Following these reviews and discussion and the timescale for implementing change was less than a week.

\section{Strategy}

PDSA cycle 1. Following the results of the initial audit we attempted to change the local guidelines for the hot clinic to then present to the consultant body at the hospital general surgical educational meeting. However, following discussion among SHOs and registrars in the department, we realised the results of the audit needed discussion to highlight what the department as a whole felt were the main issues that needed changing.

PDSA cycle 2. Therefore I arranged a consultation with consultants in the department. This occurred in a clinical governance meeting that followed the departmental educational meeting, where the whole department from FY1's to consultants are present. We presented our initial audit of the service the department was providing, the areas for improvement, and how the current service compared to the new RCS guidelines. The department discussed what needed to change, the barriers to change and how they would be overcome. As a department the suggestions for how the hot clinic should change developed, and thus all members of the team providing care had input into the planned intervention. The department identified that there was not enough senior input and communication with GPs was poor. The discussion gave a mandate for change.

PDSA cycle 3. As a result of the departmental discussion we changed local Trust guidelines to better reflect best practice as laid out by the RCS guidelines, and to reflect what the department felt was important. We changed the guideline to state that all patients must have been seen by or discussed with a registrar at some point during the patient journey prior to discharge from hot clinic, and that all patients discharged from hot clinic must have a discharge letter sent to the GP.

PDSA cycle 4. The new guidelines were printed and put in the 
handover room for the emergency surgery team and the team were informed of the new guidelines. Measurements were taken that showed no improvement in the measured outcomes.

PDSA cycle 5. The new guidelines were discussed at the following clinical governance meeting, and were agreed upon by all the consultants and juniors present. This validated the guidelines as being a mandatory part of practice. Post measurement was carried out for the four weeks following this validation.

\section{Results}

Post measurement was performed with the same method as the baseline measurement. All patients attending hot clinic from 1st August to 15th September 2014 were included $(n=32)$.

Results of this post measurement showed that:

- 34\% of patients were seen in $\mathrm{A}+\mathrm{E}$ by an $\mathrm{SHO}$ only without discussion with a senior

- Of these $34 \%, 27 \%$ were then assessed in hot clinic by an $\mathrm{SHO}$ only without senior discussion

- Therefore $9 \%$ of patients going through the hot clinic pathway never had any senior input (from 26\%)

- $63 \%$ of patients attending hot clinic had a letter sent to GP (from $18 \%)$.

The QIP demonstrates:

- A $65 \%$ decrease in patients going through the hot clinic pathway never having an senior input

- A 250\% increase in patients attending hot clinic had a letter sent to GP.

\section{Lessons and limitations}

The main challenge faced during this project was cultural change. Although the hot clinic service was acceptable and did work well for the patient, this project aimed to improve the service and had no obvious reward for the doctors taking part. It also required slightly more work from senior colleagues and a discharge letter to be written. In order to change to a new culture with more work is always difficult. However, with patient safety being of paramount importance we were confident that changes would be successful.

The essential aspect in this project was engaging the whole department right from the start. By exposing the problems in the service before trying to implement any change was essential. This allowed the seniors in the department to agree to change and suggest changes - rather than it being imposed upon them by a junior member of the department. Once the idea that change had to occur was ingrained, changing the guidelines and gaining validation was relatively simple.
The range of the changes was also small scale and could be instantly applied - therefore very quickly improving the service. These basic principles or trying to institute change can be applied to a wide range of projects. We anticipate the long term effect of the intervention to be sustained, and the local guidelines have now changed to incorporate the improvements outlined here. A major factor in maintaining the improvements is incorporating the guidelines into the new doctors induction to the department.

Limitations to the project are mainly due to variation between the doctors supplying the hot clinic service. Different SHOs and registrars run the clinic from week to week, and it is up to them to comply to guidelines. The turnover of staff that is a feature of surgical training introduces new compliance issues with every few months and refreshing the departmental knowledge and understanding of the guidelines is increasingly important as time passes.

A potential improvement would be to involve permanent staff such as nurses and ward clerks in ensuring all patients had been seen by a registrar and had a letter sent to the GP. However, a system to $100 \%$ ensure this would happen could not be put in place, there was still instances of locum staff, or annual leave whereby the correct processes could be bypassed.

\section{Conclusion}

This project has shown an increase in senior input into patients care when attending the surgical hot clinic and an improved communication with GPs, resulting in improved patient care. The project effected change by highlighting the deficits in the current service, identifying a new standard of care, discussing this with all members of the department involved with supplying the service, and then coming to a consensus to validate change. The local guidelines have now changed to incorporate the interventions, which should continue to benefit patient care. However, a sustained effort is required from the department to continue monitoring compliance to ensure they provide consistent the optimum in patient centred care.

\section{References}

1. Royal College of Surgeons of England. (2014). Commissioning Guide: Emergency General Surgery (acute abdominal pain).

2. Nuffield Trust (2013). Quality Watch report: Focus on preventable conditions.

http://www.qualitywatch.org.uk/sites/files/qualitywatch/field/fi eld_document/131010_QualityWatch_Focus_Preventable Admissions.pdf

3. Kripalani S, LeFevre F, Phillips CO, Williams MV, Basaviah $\mathrm{P}$, Baker DW. Deficits in Communication and Information Transfer Between Hospital-Based and Primary Care Physicians: Implications for Patient Safety and Continuity of Care. JAMA. 2007;297(8):831-41. doi:10.1001/jama.297.8.831. 
Declaration of interests

Nothing to declare.

\section{Acknowledgements}

Miss Stella Vig 